\title{
Testing the chemical tagging with old OC
}

\author{
Sergi Blanco-Cuaresma ${ }^{1}$, C. Soubiran ${ }^{1}$, P. Jofré ${ }^{1}$ and U. Heiter ${ }^{2}$ \\ ${ }^{1}$ CNRS / Univ. Bordeaux, LAB, UMR 5804, F-33270, Floirac, France. \\ ${ }^{2}$ Department of Physics and Astronomy, Uppsala University, Sweden
}

\begin{abstract}
We have applied the chemical tagging technique to study three old Open Clusters (NGC2632/Praesepe, NGC752 and NGC1817) by using our own spectral analysis code.
\end{abstract}

Keywords. Chemical tagging, Open Clusters, Spectroscopy

\section{Introduction}

De Silva et al. 2007 demonstrated the chemical homogeneity of two open clusters (OC) and one moving group together with the uniqueness of their abundance patterns. These findings open the possibility of using the technique of chemical tagging to identify common formation sites in the disk as proposed by Freeman \& Bland-Hawthorn 2002. We have developed our own spectral analysis code and we have applied this technique to three old OCs (NGC2632/Praesepe, NGC752 and NGC1817) observed by NARVAL.

\section{Results}

The code has been validated by using the benchmark stars' spectral library (BlancoCuaresma et al. 2013, in prep.). The parameters derived by our code are in good agreement with the reference APs (Heiter et al. / Jofré et al. 2013, in prep.) with a dispersion of $115 \mathrm{~K}$ in Teff, 0.16 dex in $\log (\mathrm{g})$ and 0.09 dex in metallicity.

We have used the synthetic spectra fitting technique and the Equivalent Width (EW) method for the abundance determination of the OCs. The former presents a general lower dispersion, making it more suitable for chemical tagging. Each cluster has different abundance patterns and the dispersion found for some elements is lower than 0.05 dex, confirming the potential of the chemical tagging technique and validating the results from previous works.
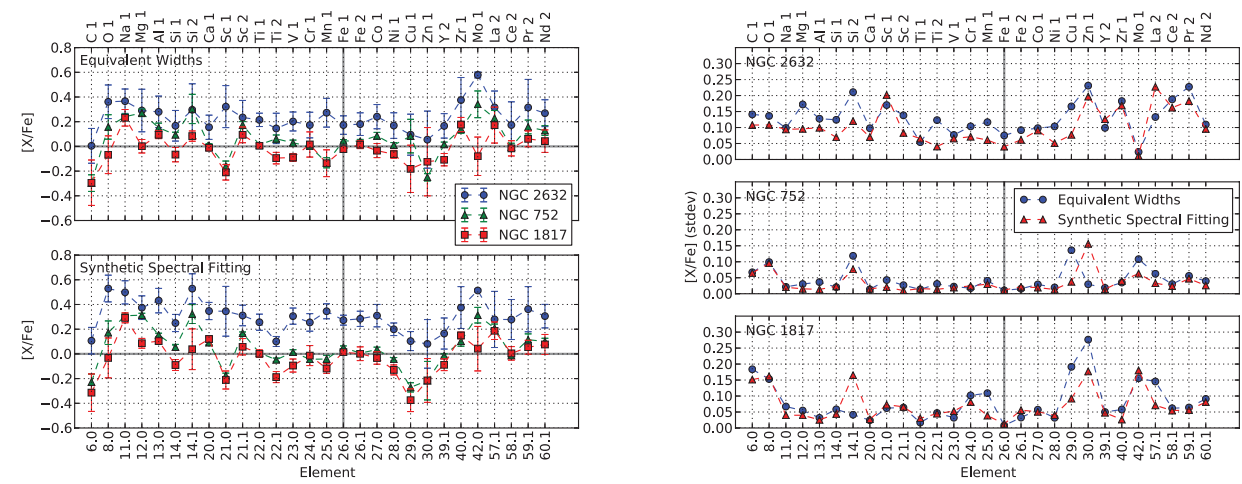

Figure 1. Abundance patterns (left) and standard deviations (right) for the analyzed OCs. 